\title{
Revealing dopant local structure of Se-doped black phosphorus
}

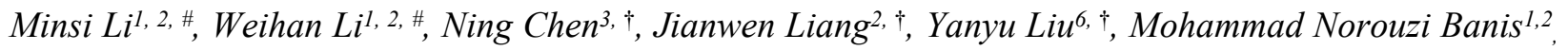
Junjie Li $^{2}$, Yiming Xiao ${ }^{1}$, Xuejie Gao ${ }^{1,2}$, Yongfeng Hü, Qunfeng Xiao ${ }^{3}$, Kieran Doyle-Davis ${ }^{2}$, Yulong Liu ${ }^{2}$, Yun Mui Yiu ${ }^{1, \dagger}$, Dejun Li ${ }^{5}$, Shiyu Liu ${ }^{5}$, Ruying Li ${ }^{2}$, Frank Brandys ${ }^{4}$, Ranjith Divigalpitiya ${ }^{4}$, Tsun-Kong Sham I, *, Xueliang Sun ${ }^{2, *}$

${ }^{1}$ Department of Chemistry and Soochow-Western Centre for Synchrotron Radiation Research, University of Western Ontario, London, Ontario, N6A 5B7, Canada

${ }^{2}$ Department of Mechanical and Materials Engineering, University of Western Ontario, London, Ontario, N6A 5B9, Canada

${ }^{3}$ Canadian Light Source, 44 Innovation Boulevard, Saskatoon, Saskatchewan, S7N 2V3, Canada ${ }^{4}$ Department of Chemistry, University of Western Ontario, London, Ontario, N6A 5B7, Canada

43M Canada Company, 1840 Oxford Street East, London, Ontario, N5V 3R6, Canada

${ }^{5}$ Tianjin International Joint Research Centre of Surface Technology for Energy Storage Materials, College of Physics and Materials Science, Tianjin Normal University, Tianjin, 300387, China.

${ }^{6}$ College of Physics and Materials Science, Tianjin Normal University, Tianjin 300387, China.

*Corresponding Author: Xueliang Sun, Tel: +1-519-661-2111 Ext 87759; Tsun-Kong Sham, Tel: +1-519661-2111 Ext 86341;

E-mail address: xsun9@uwo.ca (X. Sun); tsham@uwo.ca (T.K. Sham)

\#These authors contributed equally to this work.

$\uparrow$ These authors contributed to the theoretical study of this work. 
Table S1: Full-width-at-half-maximum of the raman peaks $\mathrm{A}_{\mathrm{g}}{ }^{1}, \mathrm{~B}_{2 \mathrm{~g}}$ and $\mathrm{Ag}_{\mathrm{g}}{ }^{2}$ of undoped $\mathrm{BP}$, SeBP-0.1, SeBP-0.2 and SeBP-0.4.

\begin{tabular}{cccc}
\hline Sample & $\mathbf{A}_{\mathbf{g}} \mathbf{1}^{\mathbf{1}}$ & $\mathbf{B}_{\mathbf{2 g}}$ & $\mathbf{A}_{\mathbf{g}}{ }^{\mathbf{2}}$ \\
\hline Undoped BP & 2.04 & 3.24 & 2.49 \\
SeBP-0.1 & 4.40 & 5.00 & 4.87 \\
SeBP-0.2 & 6.22 & 8.32 & 6.71 \\
SeBP-0.4 & 8.01 & 10.30 & 8.73 \\
\hline
\end{tabular}

Table S2. Ratio of Se-Se to Se-P peak of XPS spectra of SeBP-0.1, SeBP-0.2 and SeBP-0.4.

\begin{tabular}{|l|l|l|}
\hline & Se-Se & Se-P \\
\hline SeBP-0.1 & $62.5 \%$ & $37.5 \%$ \\
\hline SeBP-0.2 & $50 \%$ & $50 \%$ \\
\hline SeBP-0.4 & $38.2 \%$ & $61.8 \%$ \\
\hline
\end{tabular}
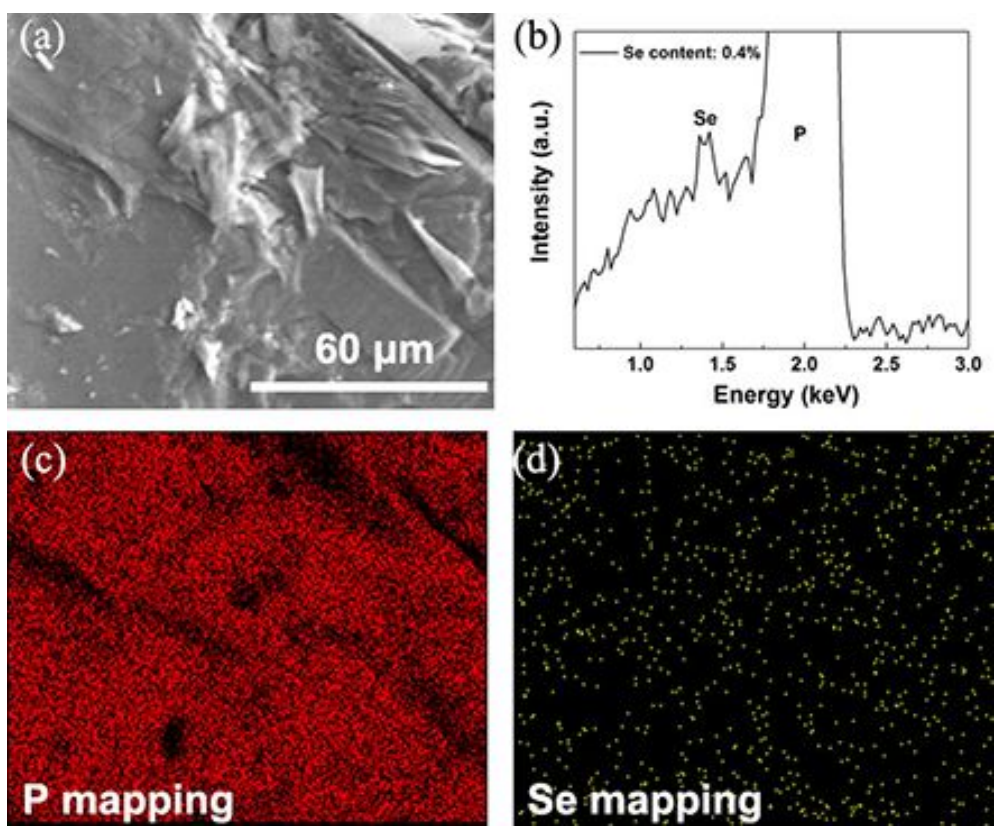

Figure S1: (a) SEM and (b) EDX spectra of SeBP-0.4. (c) and (d) are the corresponding P and Se maps in (a). 
(a)

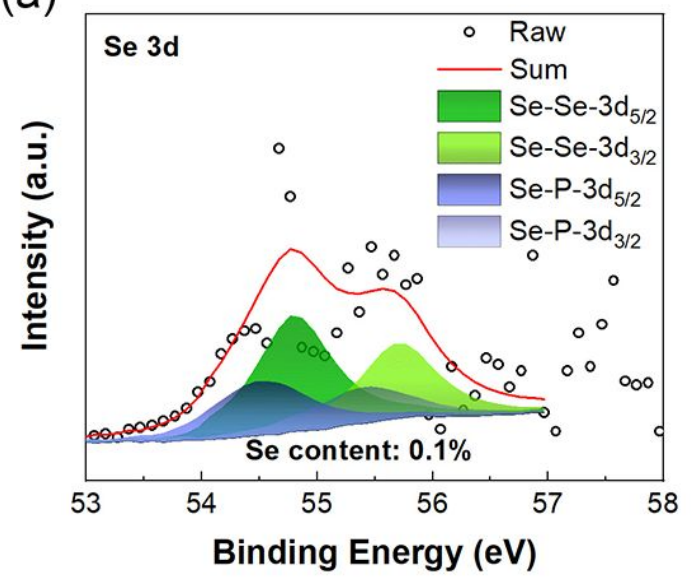

(b)

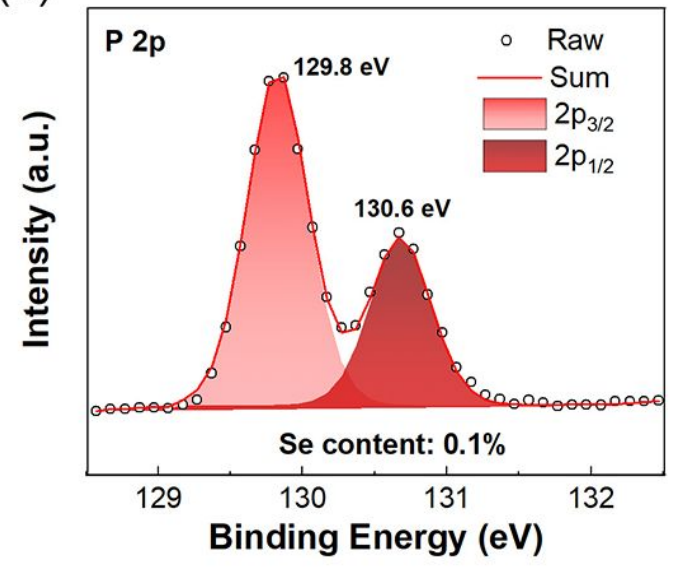

Figure S2. (a) Se 3d and (b) P 2p XPS spectra of SeBP-0.1.

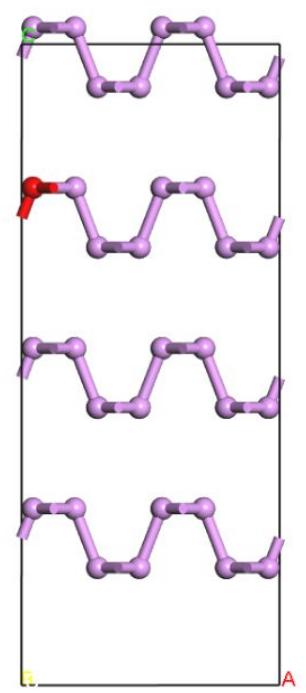

Figure S3: The model structure of $\mathrm{P}-\mathrm{Se}_{1}$ (b-axis). The $\mathrm{P}-\mathrm{Se}_{1}$ model structure is setting $0.1 \mathrm{Se}$ atom occupation in a $2 * 2 * 2$ supercell of black $\mathrm{P}\left(\mathrm{P}_{63.9} \mathrm{Se}_{0.1}\right.$, Se content is $\left.\sim 0.4 \%\right)$. 

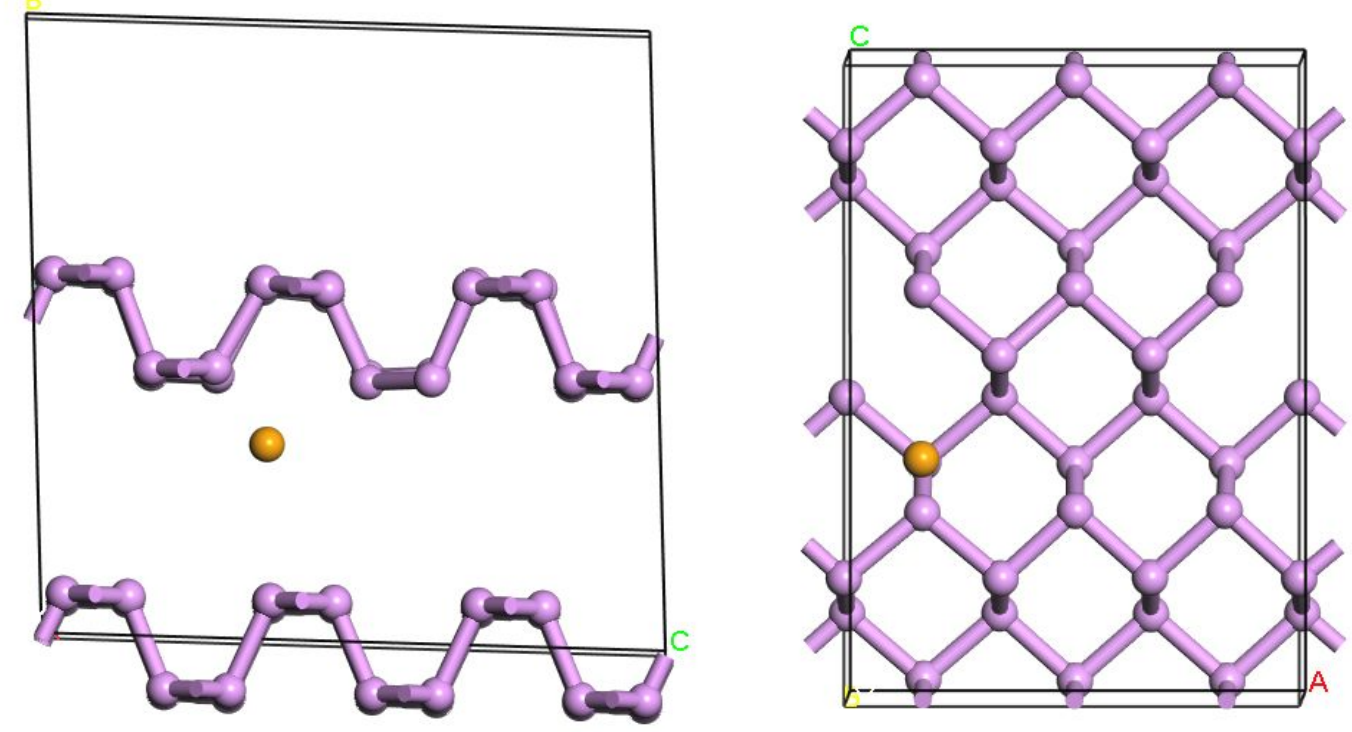

Figure S4: The model structure of $\mathrm{P}-\mathrm{Se}_{2}$ (a and b-axis). The $\mathrm{P}-\mathrm{Se}_{2}$ model structure is setting one Se atom occupant on the interlayer of a $3 * 1 * 3$ supercell of black $\mathrm{P}\left(\mathrm{P}_{72} \mathrm{Se}_{1}\right.$, Se content is $\left.\sim 1.4 \%\right)$.

(a)
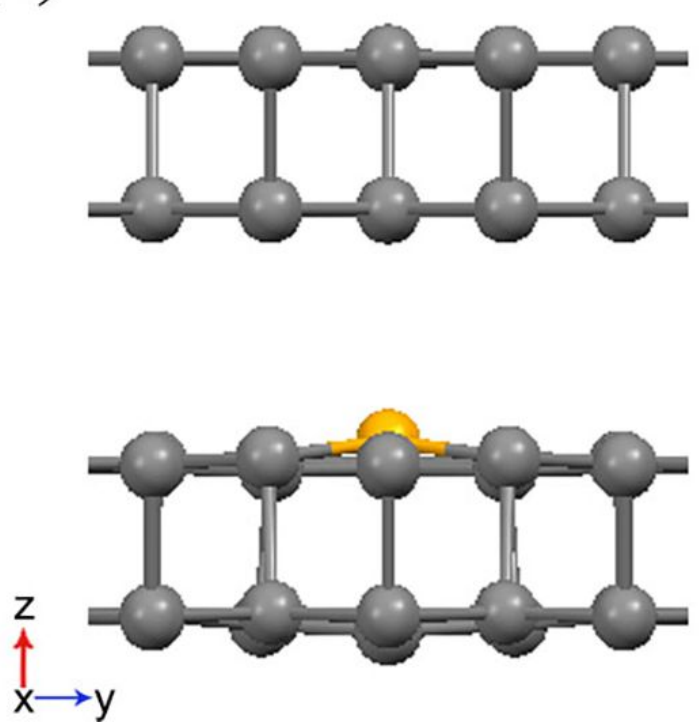

(b)

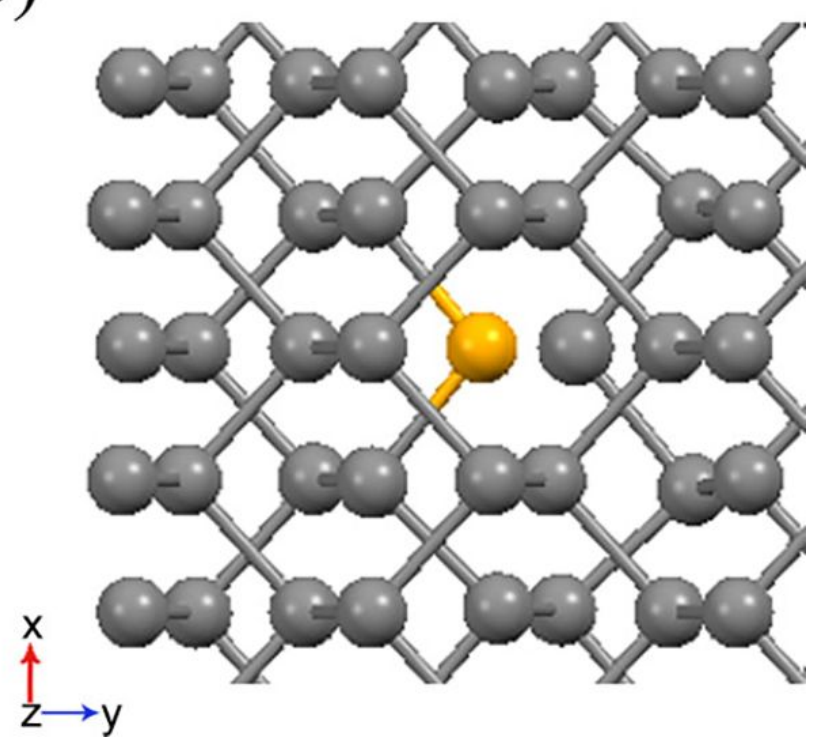

Figure S5: The (a) sideview and (b) top view model structures of $\mathrm{P}-\mathrm{Se}_{3}$. The $\mathrm{P}-\mathrm{Se}_{3}$ model structure is setting one Se atom occupant in a $3 * 1 * 3$ supercell of black $\mathrm{P}\left(\mathrm{P}_{71} \mathrm{Se}_{1}\right.$, Se content is $\sim 1.2 \%$ ). 

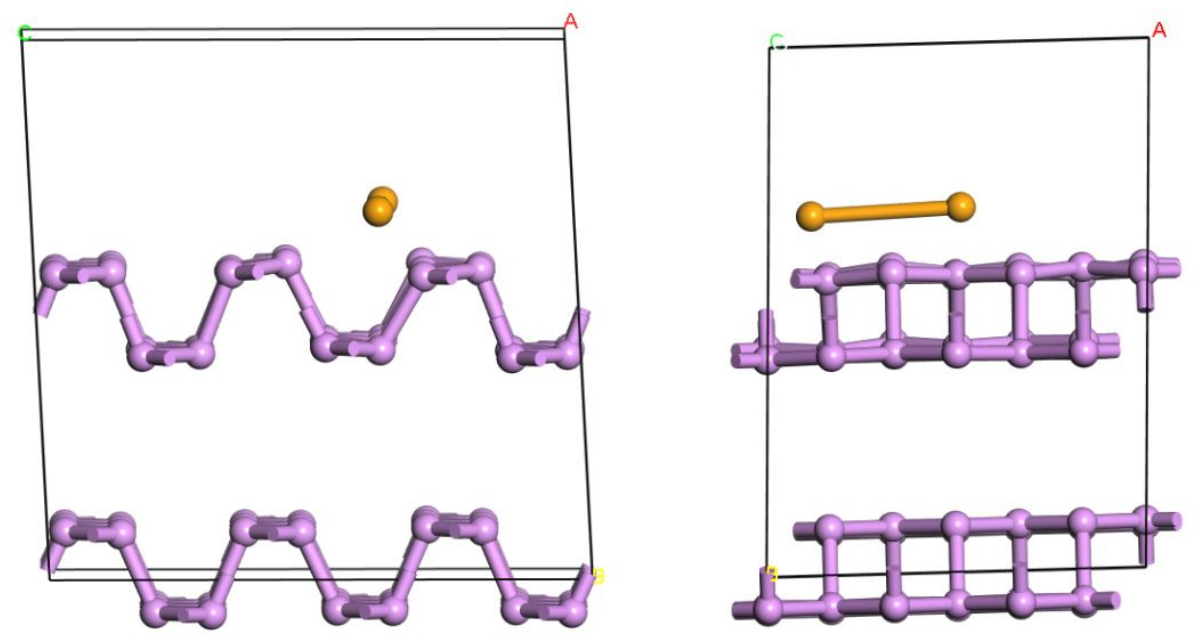

Figure S6: The model structure of $\mathrm{P}-\mathrm{Se}_{4}\left(\mathrm{a}\right.$ and c-axis). The $\mathrm{P}-\mathrm{Se}_{4}$ model structure is setting two Se atom occupant on the interlayer of a $3 * 1 * 3$ supercell of black $\mathrm{P}\left(\mathrm{P}_{72} \mathrm{Se}_{2}\right.$, Se content is $\left.\sim 2.7 \%\right)$.
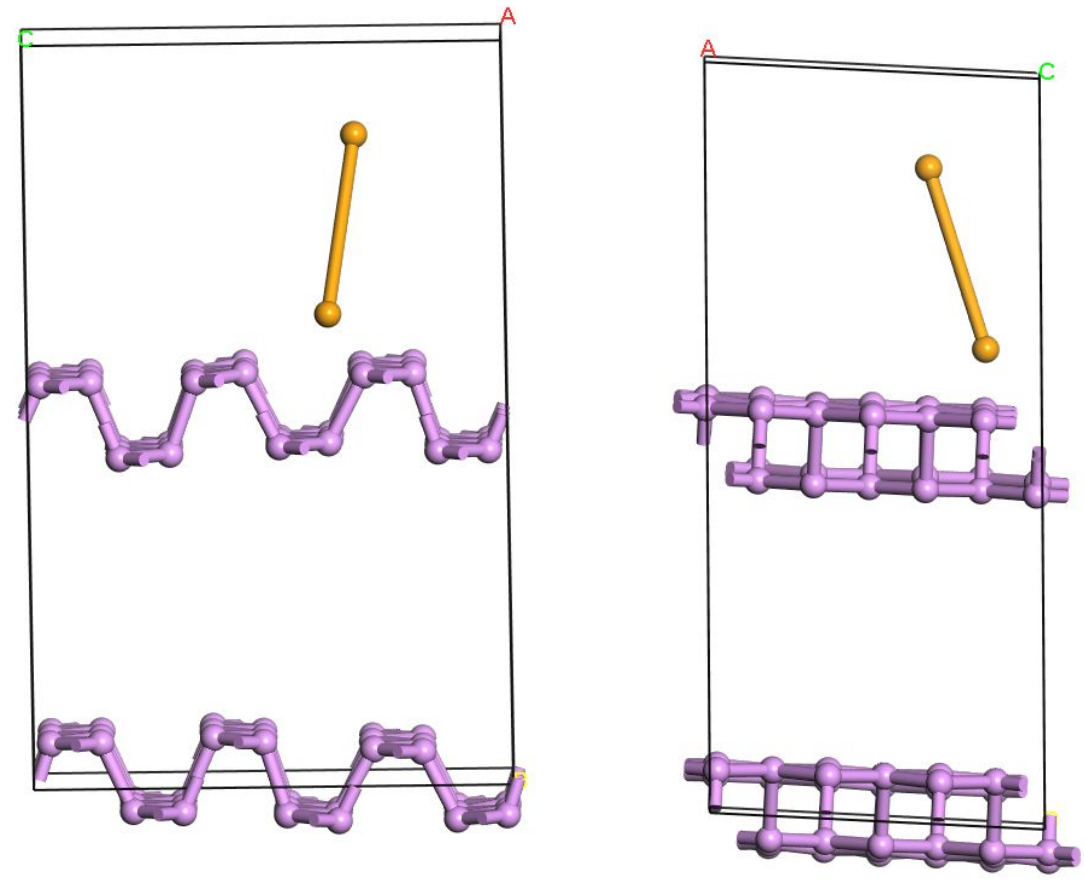

Figure S7: The model structure of $\mathrm{P}-\mathrm{Se}_{5}$ (a and c-axis). The $\mathrm{P}-\mathrm{Se}_{5}$ model structure is setting two Se atom occupant on the interlayer of a $3 * 1 * 3$ supercell of black $\mathrm{P}\left(\mathrm{P}_{72} \mathrm{Se}_{2}\right.$, Se content is $\left.\sim 2.7 \%\right)$. 

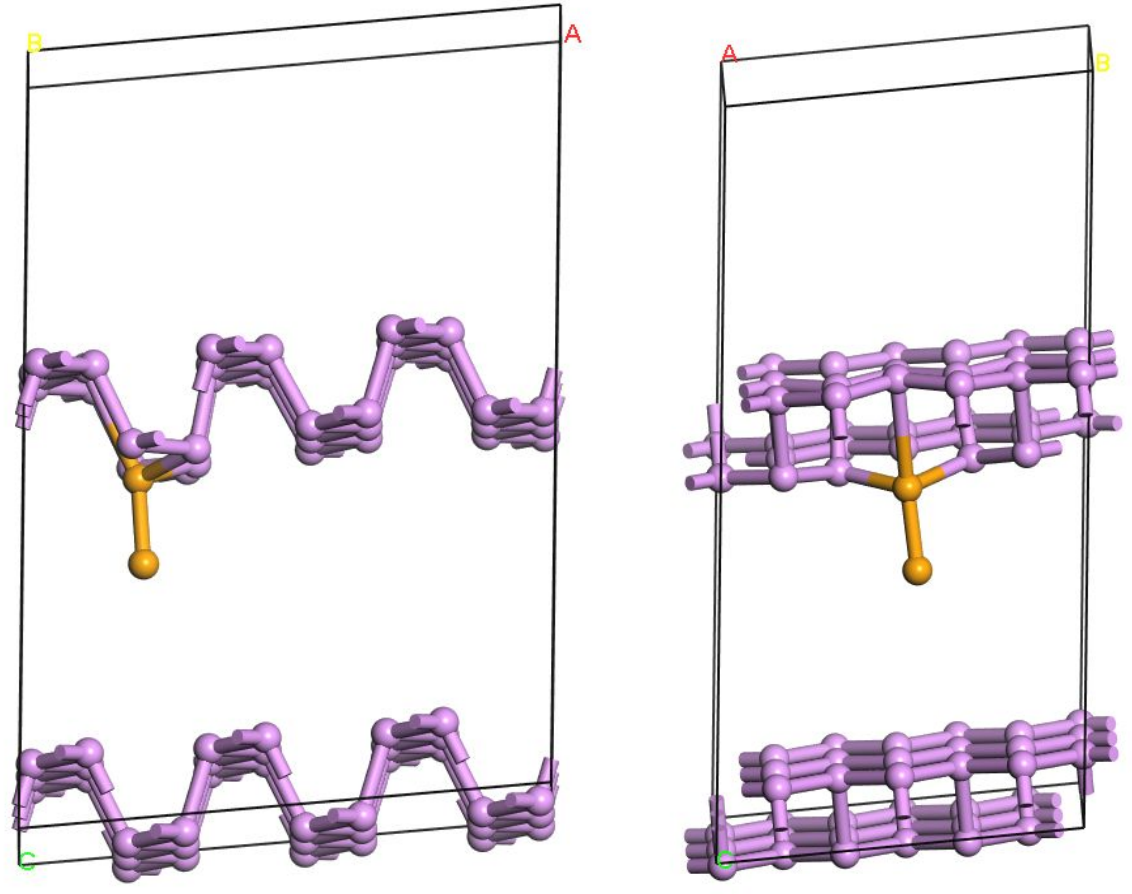

Figure S8: The model structure of $\mathrm{P}-\mathrm{Se}_{6}$ (a and c-axis). The $\mathrm{P}-\mathrm{Se}_{6}$ model structure is setting two Se atom occupant in a $3 * 1 * 3$ supercell of black $\mathrm{P}\left(\mathrm{P}_{71} \mathrm{Se}_{2}\right.$, Se content is $\left.\sim 2.7 \%\right)$. One is in the interlayer of the structure and the other one substitute the $\mathrm{P}$ site.
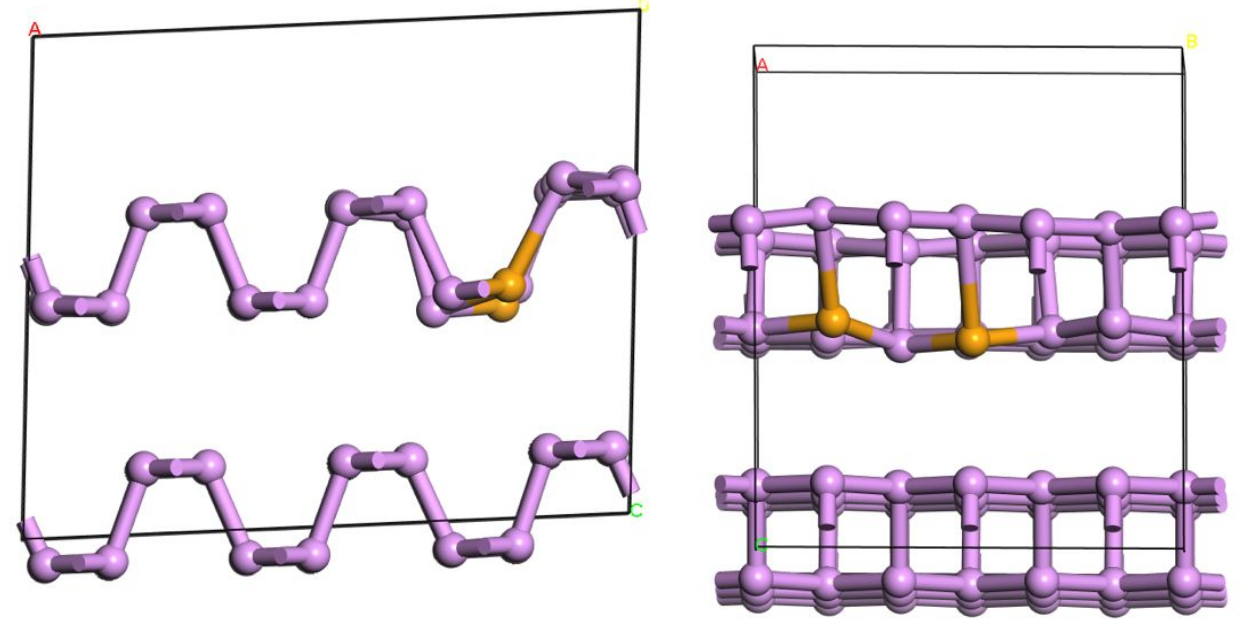

Figure S9: The model structure of $\mathrm{P}-\mathrm{Se}_{7}$ (a and c-axis). The $\mathrm{P}-\mathrm{Se}_{7}$ model structure is setting two Se atom occupant in a $3 * 1 * 3$ supercell of black $\mathrm{P}\left(\mathrm{P}_{70} \mathrm{Se}_{2}\right.$, Se content is $\left.\sim 2.8 \%\right)$. Both the Se atoms substituted the $\mathrm{P}$ site. 

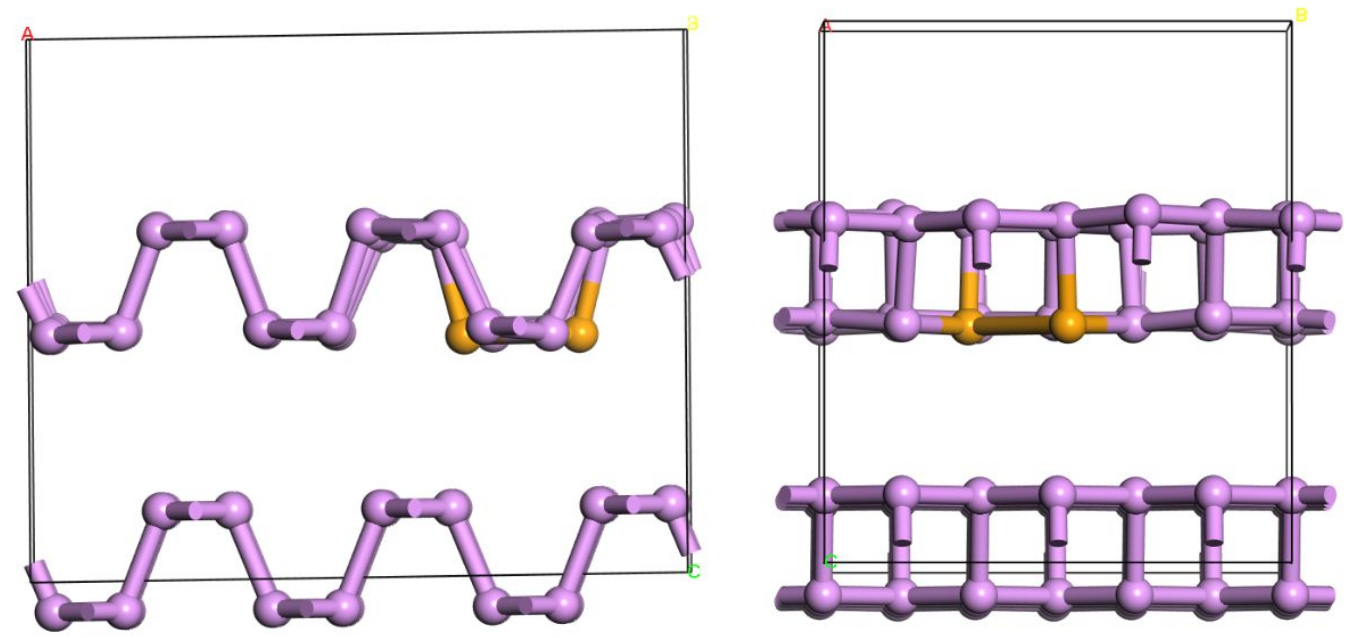

Figure S10: The model structure of $\mathrm{P}-\mathrm{Se}_{8}$ (a and c-axis). The $\mathrm{P}-\mathrm{Se}_{8}$ model structure is setting two Se atom occupant in a $3 * 1 * 3$ supercell of black $\mathrm{P}\left(\mathrm{P}_{70} \mathrm{Se}_{2}\right.$, Se content is $\left.\sim 2.8 \%\right)$. Both the $\mathrm{Se}$ atoms substituted the $\mathrm{P}$ site.
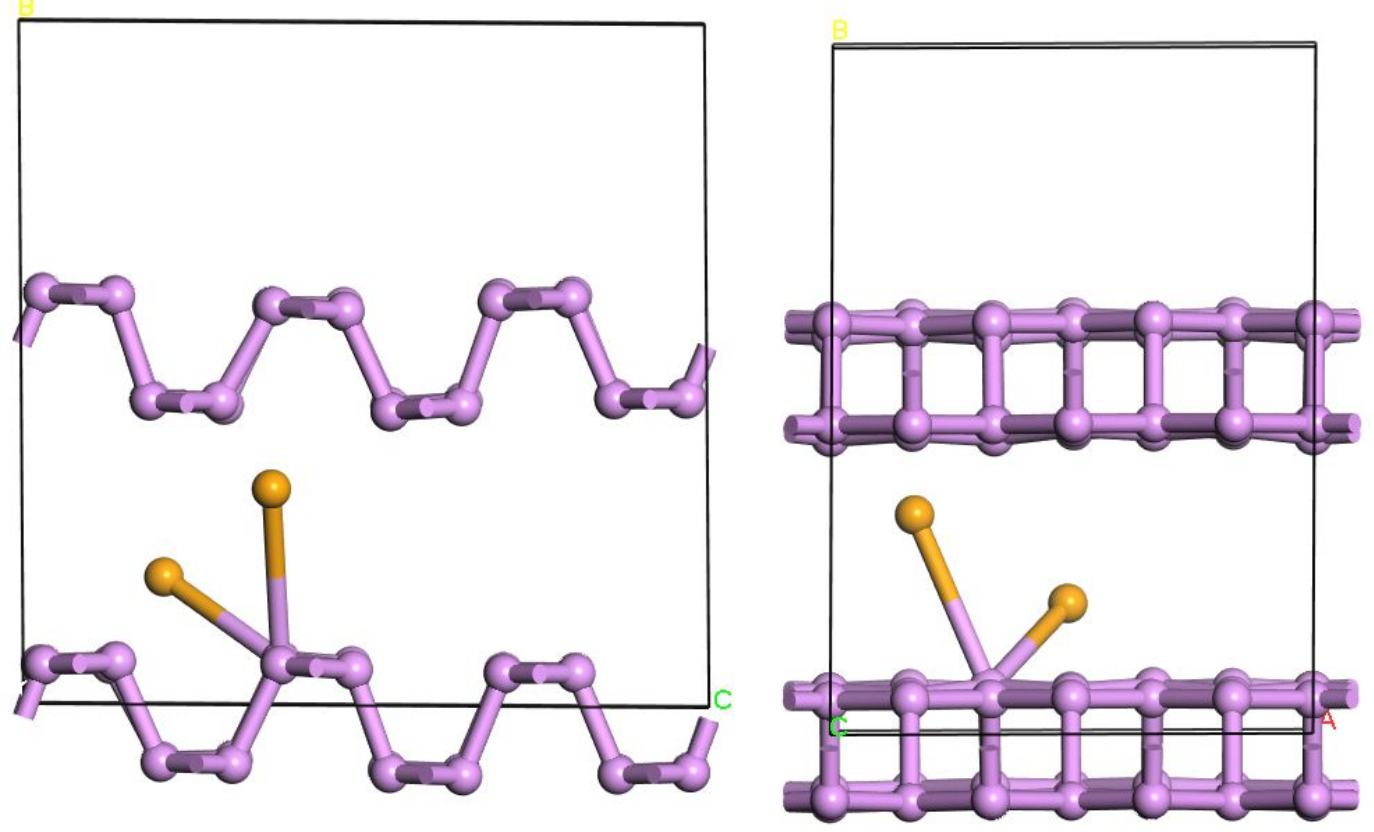

Figure S10: The model structure of $\mathrm{P}-\mathrm{Se}_{9}$ (a and c-axis). The $\mathrm{P}-\mathrm{Se}_{9}$ model structure is setting two Se atom occupant on the interlayer of a $3 * 1 * 3$ supercell of black $\mathrm{P}\left(\mathrm{P}_{72} \mathrm{Se}_{2}\right.$, Se content is $\sim 2.7 \%$ ). 

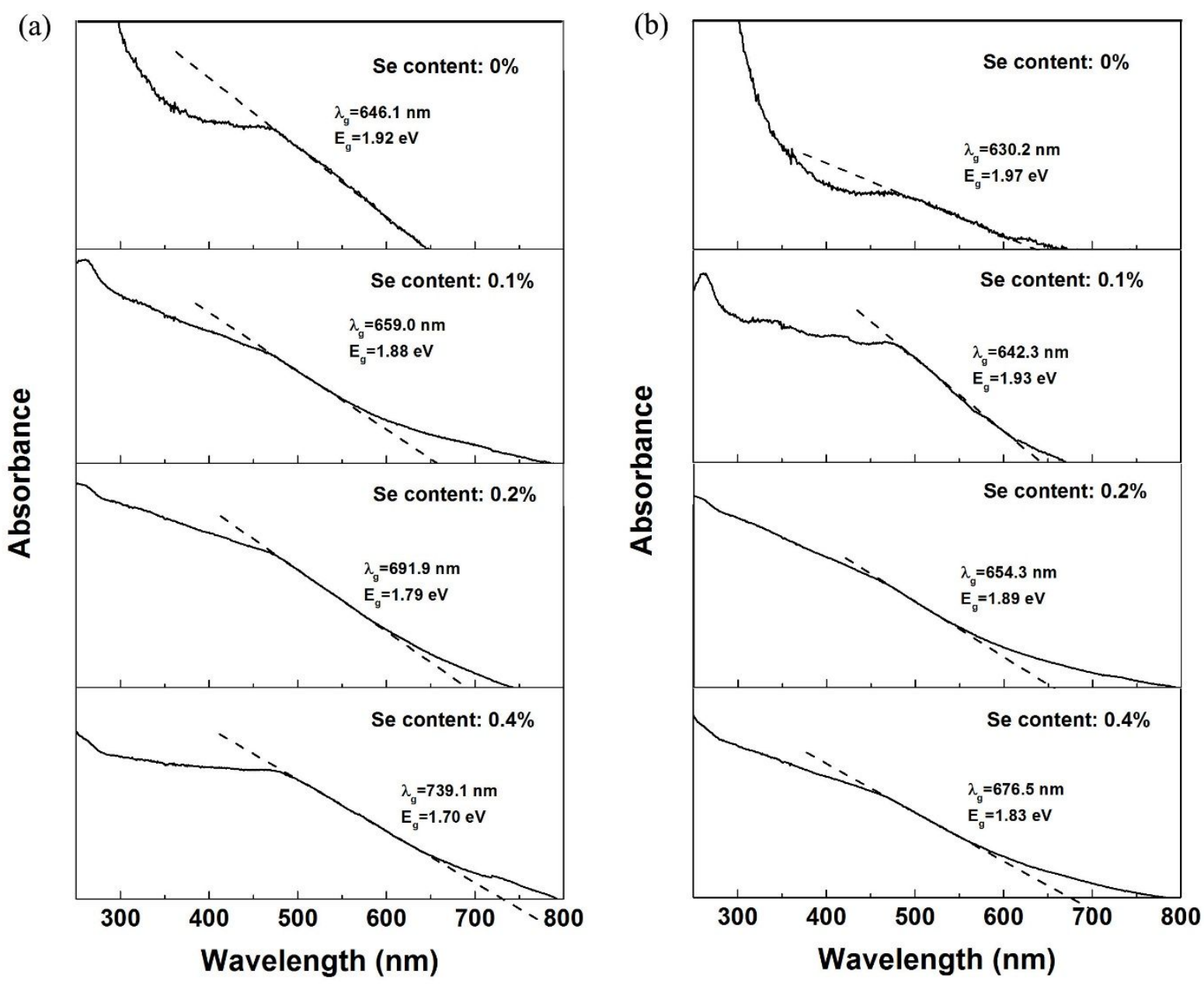

Figure S11: UV-vis absorption spectra of BP and Se-doped BP dispersed in water. Samples in (a) were not centrifuged after $9 \mathrm{~h}$ bath sonication. Samples in (b) were centrifuged under $2000 \mathrm{rpm}$ for 20 mins after $9 \mathrm{~h}$ bath sonication. 


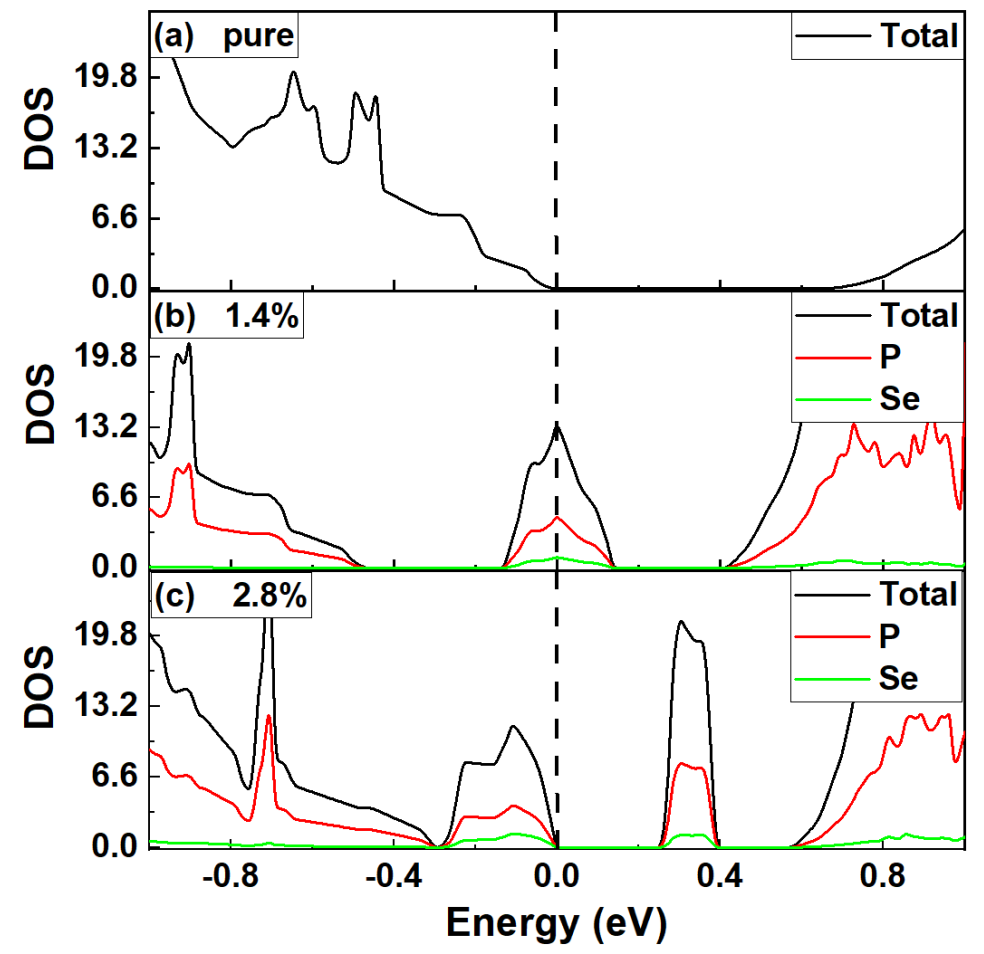

Figure S12: Total and Projected density of states (DOS) of (a) pure BP, (b) P-Se ${ }_{3}-1$, Se content is $\sim 1.4 \%$ and (c) $\mathrm{P}_{-} \mathrm{Se}_{3}-2$, Se content is $\sim 2.8 \%$. 